\title{
La Réforme et la fable, dir. A. VINTENON et F. POULET
}

\section{Maurizio Busca}

\section{OpenEdition \\ Journals}

\section{Edizione digitale}

URL: https://journals.openedition.org/studifrancesi/43709

DOI: 10.4000/studifrancesi.43709

ISSN: 2427-5856

\section{Editore}

Rosenberg \& Sellier

\section{Edizione cartacea}

Data di pubblicazione: 1 juin 2021

Paginazione: 206-207

ISSN: 0039-2944

\section{Notizia bibliografica digitale}

Maurizio Busca, «La Réforme et la fable, dir. A. vintenon et f. poulet», Studi Francesi [Online], 193 (LXV | I) | 2021, online dal 01 juillet 2021, consultato il 15 octobre 2022. URL: http://journals.openedition.org/ studifrancesi/43709 ; DOI: https://doi.org/10.4000/studifrancesi.43709

Questo documento è stato generato automaticamente il 15 octobre 2022.

\section{(c)}

Creative Commons - Attribuzione - Non commerciale - Non opere derivate 4.0 Internazionale - CC BYNC-ND 4.0

https://creativecommons.org/licenses/by-nc-nd/4.0/ 


\title{
La Réforme et la fable, dir. A. VINTENON et F. POULET
}

\author{
Maurizio Busca
}

\section{NOTIZIA}

La Réforme et la fable, dir. A. VINTENON et F. POULET, Genève, Droz, 2018, «Cahiers

d'Humanisme et Renaissance» 155, 517 pp.

1 Secondo una vulgata critica ancora diffusa, nel corso del Cinquecento le opere di finzione sarebbero state oggetto di condanna sistematica in ambito protestante. Per lungo tempo, in realtà, la materia fabulosa - sia essa letteraria, drammatica o figurativa - non è stata semplicemente rigettata: al contrario, numerose e significative sono state le operazioni di appropriazione e di utilizzo a fini parenetici e di propaganda. E, come ricorda Frank Lestringant nella prefazione al presente volume, se nel corso degli anni '70 del secolo si assiste alla proscrizione formale del teatro e, più generalmente, all'irrigidimento delle posizioni riformate circa la legittimità dell'uso dell'immagine e della finzione, nei decenni successivi l'eredità dell'approccio riformato alla fable sarà in certa misura accolta nel mondo cattolico. Prendendo in esame un corpus che si estende dall'inizio del secolo XVI ai primi anni del secolo successivo, gli atti del convegno di Bordeaux del 10-11 dicembre 2015 esplorano da un lato il (meta)discorso riformato sulla fable, sulla sua interpretazione allegorica e sul suo impiego nella scrittura poetica, dall'altro il ricorso alla fable come strumento di polemica, propaganda e conversione. Il prezioso intervento liminare di Vintenon e Poulet traccia un bilancio critico delle questioni affrontate nel volume e introduce i diciannove contributi seguenti, articolati in cinque sezioni tematiche.

2 Frank LESTRINGANT, Préface, pp. 9-15; Françoise POULET et Alice VINTENON, Introduction, pp. 17-60; Première partie («Les commentaires réformés des fictions païennes»): Isabelle PANTIN, Melanchthon et les fables païennes, pp. 63-78; céline BOHNERT, Ovidii (ut ita dicam) Chronicon: sur les textes liminaires de la "Fabularum Ovidii Interpretatio" de Georg Schuler 
(1555), pp. 79-100; Antoine BISCÉRÉ, Dresser un «tabernacle» pour Ésope. Le prestige de la fable ésopique dans les cercles protestants allemands, pp. 101-123; christiane DELOINCE-LOUETTE, Quel statut pour la fable d'Homère? Quelques lectures de commentateurs réformés à la fin du XVI e siècle, pp. 125-140; Ruth STAWARZ-LUGINBÜHL, "La Vie de Cyrus le Grand" selon Calvin: une fiction historico-théologique, pp.141-161; Deuxième partie («Fictions évangéliques»): Nicolas LE CADET, "Ce sont beaux textes d'evangile en francoys»: la place de la Bible dans "Pantagruel" (ch. xxx), pp. 165-181; Adeline DESBoIS-IENTILE, La fantasie de François Habert: variations poétiques sur le jugement de Pâris, pp. 183-200; Troisième partie («La perception de la mythologie: autour de Simon Goulart»): Teresa CHEVROLET, «Nomine mutato, narratur fabula de te»: la mythologie d'après le "Theatre du monde" de Simon Goulart, pasteur genevois, pp. 203-240; olivier РОт, De la fable à l'emblème: la «mythologie blanche» de Simon Goulart, pp. 241-272; Natacha SALLIOT, Appartenance confessionnelle et statut de la fable dans les commentaires de "La Sepmaine" de Du Bartas (1581-1585), pp. 273-293; Quatrième partie («Pédagogie et édification»): Mathilde BERNARD, Mythologie, fable et emblème, ou l'ambiguïté du rapport à la fiction chez Guillaume Guéroult, pp. 297-308; Mathieu DE LA GORCE, Se réformer avec Ovide. La "Métamorphose chrétienne" de Pierre Viret, pp. 309-332; Padraic LAMB, L'appât de la fable: dieux paiens et prosélytisme dans les traités dévots de Stephan Batman, ministre anglican, pp. 333-351; Inès KIRSCHLEGER, Le loup et l'agneau dans la prédication réformée du XVII siècle, ou comment combattre la raison du plus fort?, pp. 353-371; Christabelle THOUIN-DIEUAIDE, L'utilisation de la fable dans les sermons réformés de la première moitié $d u$ xvII ${ }^{\mathrm{e}}$ siècle, pp. 373-388; Cinquième partie («La place de la fable dans la poésie évangélique et réformée»): Nadia CERNOGORA, Persistance de la fable dans la poésie protestante: l'exemple des "Hieropoemes" de Loys Saunier (1584), pp. 391-418; Gilles couffignal, Des fables antiques à la fable de la langue: Pey de Garros, poète protestant et gascon, pp. 419-436; Audrey DURU, La fonction fable et les “Cuvres" d'André Mage de Fiefmelin (Saintonge, 1601), pp. 437-455; Adrienne PETIT, La relégation des fables et de l'«antiquaille» dans le roman sentimental, un topos post-tridentin, pp. 457-474.

3 La prima parte del volume è dedicata alle pratiche ermeneutiche e ai commenti alle fabulae pagane. PANTIN e BOHNERT si interessano rispettivamente a Melanchthon e a Sabinus, entrambi difensori della letteratura antica. Per i due umanisti il mito classico è un efficace strumento didattico, attraverso cui è possibile dispensare insegnamenti di natura morale (oltre che poetica e retorica), pur senza ricorrere alla pratica dell'allegoresi o ad una lettura filtrata attraverso il prisma dei testi sacri. BISCÉRÉ e DELOINCE-LOUETTE si concentrano invece intorno alla ricezione di Esopo e Omero. Se il primo conosce un successo notevole negli ambienti riformati e l'approvazione dello stesso Lutero in virtù della portata morale e pedagogica delle sue favole, il secondo è oggetto di giudizi più prudenti: i commentatori tendono infatti a rinunciare all'interpretazione della fabula per guardare essenzialmente alla dimensione poetica e retorica dei poemi omerici. STAWARZ-LUGINBÜHL, infine, esamina il caso dell'elaborazione, da parte di Calvino, di una vita di Ciro il Grande che attinge sia ai dati forniti dalle fonti veterotestamentarie che a quelli provenienti dalle fonti storiografiche, e che intende fare della narrazione storica una lezione paradigmatica dell'azione provvidenziale. I contributi di LE CADET e DESBOIS-IENTILE, riuniti nella seconda parte, guardano a due autori non riformati ma di sensibilità evangelica, Rabelais e Habert. Rabelais inserisce nel cap. XXX di Pantagruel una visione infernale in cui si intrecciano molteplici fonti antiche, medievali e moderne: la complessità del gioco intertestuale invita a non ridurre tale episodio a una semplice contaminazione fra il modello della catabasi epica e il motivo 
evangelico della caduta dei potenti, ma a interpretarlo alla luce di una pluralità di dimensioni complementari. Anche l'approccio di HABERT al trattamento della finzione letteraria si rivela complesso, per non dire contraddittorio: la tensione fra l'interesse per il mito e l'associazione di quest'ultimo all'errore della religione pagana (e, di conseguenza, la ricerca di una sintesi fra il messaggio evangelico e la fabula antica) è studiata attraverso l'analisi delle rappresentazioni poetiche di Atena, Giunone e Venere. La terza parte del volume è interamente dedicata alla figura di Simon Goulart, la cui considerazione della mitologia greco-latina è illustrata soprattutto alla luce dell'analisi del Theatre du monde e del commento alla Sepmaine di Du Bartas. CHEVROLET, POT е SALLIOT mostrano come, al di là della condanna del paganesimo, Goulart riconosca il valore esemplare delle fabulae, la loro efficacia a fini parenetici e la parte di verità che queste contengono in quanto espressione di una filosofia naturale: «[la mythologie] est le point d'articulation, dans l'épopée de la Création, entre la science abstraite et l'expérience sensible, entre le savoir sur le monde [...] et la perception merveilleuse, poétique et esthétique [...] qu'on en a sur le plan des phénomènes» (Pot, p. 272). La quarta parte è incentrata sul ricorso alla finzione a fini pedagogici ed edificanti. BERNARD esamina la posizione ambigua di Guillaume Guéroult, autore, traduttore ed editore critico nei confronti della mitologia ma conscio del suo valore didattico e della sua efficacia come vettore delle idee della Riforma, veicolate attraverso l'esplicitazione sistematica della morale. Dinamiche affini sono rilevate da DE LA GORCE, che si concentra sulla Métamorphose chrétienne di Pierre Viret: anche in questo caso, il ricorso al modello classico - in primo luogo ovidiano - e alla finzione mitologica appare funzionale alla promozione di un percorso di trasformazione morale e spirituale. Tali analisi trovano echi puntuali, per quanto riguarda l'ambito inglese e quello francese del Seicento, nei saggi di LAMB, KIRSCHLEGER e THOUIN-DIEUAIDE. La quinta e ultima sezione del volume prende in esame una serie di casi di impiego della fable nella letteratura evangelica e riformata, indagando in particolare le incongruenze e gli scarti fra il metadiscorso autoriale sulla finzione fabulosa e la scrittura poetica. Se CERNOGORA, COUfFIGNAL e DURU rilevano un approccio problematico - quando non contraddittorio - nella pratica dei poeti Loys Saunier, Pey de Garros e André Mage de Fiefmelin, che condannano la fable ma vi fanno regolarmente ricorso (benché per ragioni e secondo modalità differenti), PETIT segnala come, per quanto riguarda la produzione romanzesca, tra la fine del Cinquecento e l'inizio del secolo successivo si osservino delle dinamiche opposte: un autore cattolico come Antoine de Nervèze rifiuta apertamente le invenzioni antiche, mentre un suo imitatore protestante come Nicolas Des Escuteaux arriva a rivendicare apertamente un rapporto di filiazione con la letteratura classica. 